\title{
NOTIFICAÇÃO DE QUEDA DO PACIENTE CIRÚRGICO ANTES E APÓS TREINAMENTO EM SERVIÇO*
}

\author{
Suelen Reiniack ${ }^{1}$, Cristiane Ferraz da Silva ${ }^{2}$, Marisol da Paz ${ }^{3}$, Karinne Cristinne da Silva Cunha ${ }^{4}$
}

\begin{abstract}
RESUMO: Estudo tipo antes-depois com objetivo de identificar registros e perfil de queda de paciente, executar e avaliar uma tecnologia educacional. Realizado no período de abril de 2015 a fevereiro de 2016, em uma unidade de internação cirúrgica de um hospital público no município do Rio de Janeiro. Verificou-se o registro de uma queda antes do treinamento (33,3 quedas por 1.000 paciente-dia) no período de setembro de 2015. Após um período de seis meses, verificou-se o registro de seis quedas notificadas adequadamente $(162,2$ quedas por 1.000 paciente-dia). Destas, quatro do sexo masculino possuíam em média 57,2 anos de idade, quatro estavam orientados, quatro foram da própria altura, uma do leito e uma da cadeira, sendo que duas obtiveram dano. Observou-se falha de notificação e prováveis subnotificações. Não há rotina de prevenção e nem formas de capacitação continuada, sendo imprescindível a implantação da escala de avaliação do risco de queda na instituição.
\end{abstract}

DESCRITORES: Enfermagem; Tecnologia educacional; Educação em saúde; Segurança do paciente.

\section{REPORTING OF INPATIENT FALLS BEFORE AND AFTER IN-SERVICE TRAINING}

\begin{abstract}
Before-after study aimed to identify the records and the profile of inpatient falls, promote and assess an Educational Technology conducted from April 2015 to February 2016, in the surgery center of a public hospital in Rio de Janeiro. Prior to in-service training one fall was properly recorded (33.3 falls per 1,000 patients-day) in September 2015. After a six-month period, it was found that six falls of inpatients were properly reported ( 162.2 falls per 1,000 patients-day). Of these patients, four were male individuals, with a mean age of 57.2 years, four were oriented, four fell down from a standing position, one patient fell from the bed andone fell from a chair. Also, two falls caused harm. Lack of reporting and probable underreporting was observed. There is no routine of prevention and no permanent training of the staff, and it is absolutely necessary to implement a scale of assessment of risk of falls in the institution.
\end{abstract}

DESCRIPTORS: Nursing; Educational technology; Health education; Patient safety.

\section{NOTIFICACIÓN DE CAÍDA DEL PACIENTE QUIRÚRGICO ANTES Y DESPUÉS DE CAPACITACIÓN EN SERVICIO}

RESUMEN: Estudio tipo antes-después objetivando identificar registros y perfil de caída de pacientes, ejecutar y evaluar una tecnología educativa. Realizado entre abril de 2015 y febrero de 2016, en unidad de internación quirúrgica de hospital público del municipio de Rio de Janeiro. Se verificó el registro de una caída antes de la capacitación (33,3 caídas cada 1000 pacientes-día) durante setiembre de 2015. Pasado un período de seis meses, se verificó registro de seis caídas correctamente notificadas (162,2 caídas cada 1000 pacientes-día). De estas, cuatro del sexo masculino, media etaria de 57,2 años, cuatro estaban orientados, cuatro fueron desde la propia altura, una desde el lecho, y una desde la silla, ambas con daño. Se observó falta de notificación y probables subnotificaciones. No existe rutina de prevención ni formas de capacitación continua, resultando imprescindible la implantación de la escala de evaluación del riesgo de caída en la institución.

DESCRIPTORES: Enfermería; Tecnología Educacional; Educación en Salud; Seguridad del Paciente.

*Artigo extraído da dissertação intitulada: "Tecnologia educacional para prevenção de queda do paciente cirúrgico baseada na análise dos fatores de risco". Universidade Federal do Estado do Rio de Janeiro, 2016.

${ }^{1}$ Enfermeira. Discente de Especialização em Clínica Médica e Cirúrgica. Hospital Federal da Lagoa. Rio de Janeiro, RJ, Brasil. Enfermeira. Mestre em Saúde e Tecnologia no Espaço Hospitalar. Universidade Federal do Estado do Rio de Janeiro. Rio de Janeiro, RJ, Brasil.

${ }^{3}$ Enfermeira. Especialista em Qualidade e Segurança em Saúde. Hospital Federal da Lagoa. Rio de Janeiro, RJ, Brasil.

${ }^{4}$ Enfermeira. Doutora em Neuroimunologia. Docente de Enfermagem e Pós-graduação em Saúde e Tecnologia no Espaço Hospitalar. Universidade Federal do Estado do Rio de Janeiro. Rio de Janeiro, RJ, Brasil.

Autor Correspondente:

Recebido: $11 / 07 / 2016$

Suelen Reiniack

Finalizado: 30/01/2017

Hospital Federal da Lagoa

R. Mena Barreto, 151 - 22.271.100 - Rio de Janeiro, RJ, Brasil

E-mail: sukaa.r@gmail.com 


\section{- INTRODUÇÃO}

A preocupação com a qualidade e segurança do paciente nos serviços de saúde aumentou, sendo que metade dos incidentes é evitavel, e podem ter impacto negativo sobre os pacientes e seus familiares, instituições e sociedade. Com a hospitalização, aumenta-se o risco de queda pelo fato dos pacientes se encontrarem em ambientes não familiares, serem portadores de doenças que predispõem à queda, sendo também submetidos aos múltiplos procedimentos terapêuticos e múltiplas prescrições medicamentosas ${ }^{(1-2)}$.

Para o monitoramento e prevenção de danos na assistência à saúde, no ano de 2013 foi instituído pela Portaria n. 529 o Programa Nacional de Segurança do Paciente. Juntamente com a criação de seis Metas Internacionais de Segurança do Paciente pela Organização Mundial de Saúde (2013), dentre elas a Meta Seis: redução do risco de lesões ao paciente em decorrência de queda, que também tornou compulsória a notificação de Eventos Adversos (EA) pelos serviços de saúde ${ }^{(3)}$.

No mesmo ano o Ministério da Saúde criou o Protocolo de Prevenção de Quedas, com o objetivo de reduzir a ocorrência de quedas e danos, através da implementação de medidas que contemplem a avaliação de risco do paciente, garantam o cuidado multiprofissional, um ambiente seguro, e promovam a educação do paciente, familiares e profissionais ${ }^{(2)}$.

O conceito de Queda é o deslocamento não intencional do corpo para um nível inferior à posição inicial, resultando ou não em dano. Também, quando o corpo é encontrado no chão ou durante o deslocamento, necessita de amparo, ainda que não chegue ao chão ${ }^{(2)}$.

A segurança do paciente visa à redução do risco de danos desnecessários relacionados com os cuidados de saúde, para um mínimo aceitável, sendo o dano abordado como comprometimento da estrutura ou função do corpo, podendo ser físico, social ou psicológico. Por outro lado, o incidente é uma circunstância que resulta, ou não, em dano desnecessário ao paciente. Quando este resulta em dano ao paciente, é nomeado $\mathrm{EA}^{(2)}$.

É extremamente importante adotar indicadores e monitoramento de processos para reduzir o impacto dos danos nos sistemas de saúde, pois a queda pode gerar impacto negativo sobre a mobilidade dos pacientes, além de ansiedade, depressão e medo de cair novamente, contribuindo para o aumento do tempo de internação e custos assistenciais. Portanto, além da implantação de novas rotinas e protocolos, é necessário desenvolver estratégias educativas como mecanismos centrais para a divulgação e compromisso com a cultura de segurança ${ }^{(1-2)}$.

Diante disto, um exemplo de estratégia educativa é a Tecnologia Educacional (TE), caracterizada como fundamento filosófico voltado para o desenvolvimento do indivíduo e caracterizado por novas teorias, ensinos, pesquisas, conceitos, técnicas para a atualização da educação, facilitando o aprendizado e contribuindo para o avanço educacional. Não se limita apenas ao uso de equipamentos, pois está voltada para a organização lógica das atividades sistematicamente observadas, compreendidas e socializadas $^{(4)}$.

Em outro aspecto, as instituições devem facilitar a comunicação de incidentes através de notificações de EA em formulários próprios, pois são importantes ferramentas de informação ao Núcleo de Segurança do Paciente (NSP), sendo que as notificações têm como objetivo principal o aumento da segurança do paciente.

O NSP da instituição hospitalar do presente estudo foi criado em 2014, que segue seu próprio protocolo de quedas criado no ano de 2013, sendo constituído por uma Enfermeira responsável pelas ações de melhoria da segurança do paciente, como a investigação dos EA notificados no formulário institucional.

O NSP da instituição apontava para a baixa utilização da ficha específica para notificação de EA, sendo utilizado apenas o livro de ordens e ocorrências para registro de queda. Com base nesses dados, escolheu-se como objeto de estudo a notificação de queda do paciente.

Esta pesquisa tem como objetivos identificar o perfil e registros de queda em uma unidade de internação cirúrgica; executar e avaliar uma TE a respeito da notificação de queda de paciente. 


\section{METODOLOGIA}

Trata-se de um estudo do tipo antes-depois, de abordagem quantitativa. Realizado no período de abril de 2015 a fevereiro de 2016, em uma unidade de internação cirúrgica de um hospital público no município do Rio de Janeiro. Nesta unidade de internação cirúrgica estão incluídas as clínicas de Cirurgia Geral, Vascular, Proctologia e Neurocirurgia. Sendo caracterizadas como pré-operatório e pós-operatório de cirurgias eletivas, com capacidade para 28 leitos, atendendo pacientes do Sistema Único de Saúde (SUS) acima de 18 anos de idade.

A instituição não adota nenhuma escala em particular para avaliar o risco de queda, tem como foco intervenções de prevenção norteadas pelo risco de queda utilizando pulseira de cor amarela no momento da internação nos pacientes menores de cinco anos, maiores de sessenta e cinco anos, confusos, agitados, com distúrbio neurológico, sedados ou com dificuldade de marcha.

A amostra foi composta por todos os registros de quedas ocorridos no período de abril de 2015 a fevereiro de 2016 no setor de internação cirúrgica. Para obtenção do índice de queda, foram considerados os registros na ficha específica de notificação de EA do NSP da instituição, e também nas anotações dos profissionais de enfermagem registradas no livro ata de ordens e ocorrência.

Durante a coleta de dados, houve a necessidade de construir uma TE sobre a temática prevenção de quedas do paciente, com foco no processo de notificação do evento para os profissionais de enfermagem do cenário do estudo. Sendo assim, no mês de setembro de 2015, o serviço de Educação Permanente em conjunto com o NSP realizaram o treinamento em serviço para os profissionais de enfermagem, a partir da TE construída pelas autoras deste estudo.

A TE foi planejada segundo um plano de aula, contendo as seguintes etapas: tema abordado, justificativa, objetivos, metodologia, bibliografia, previsão de tempo, plano para avaliação posterior juntamente com registros e formulários de notificação das quedas. Sendo assim, a TE foi construída em forma de apresentação multimídia no Windows Power Point 2013, contendo 30 slides ilustrativos e autoexplicativos.

O conteúdo selecionado abordou as categorias baseadas no Protocolo de Prevenção de Quedas do Ministério da Saúde (MS) de 2013: Programa Nacional de Segurança do Paciente; Protocolo de Prevenção de Quedas; Definição de conceitos como Queda, Incidente, Dano e EA; Fatores contribuintes Extrínsecos e Intrínsecos; Prevenção baseada nos fatores de risco com medidas Universais e Individuais; Notificação e fluxograma da notificação da instituição hospitalar ${ }^{(2)}$.

A aplicação da TE foi realizada no início do mês de setembro de 2015 no próprio local e horário de trabalho, por meio de uma aula expositora dialogada, articulando informações e considerando os conhecimentos prévios dos profissionais. Foi necessário um encontro de 45 minutos por turno no período de três dias e três noites para abranger todos os plantões. Participaram ativamente o total de 38 profissionais Enfermeiros e Técnicos de Enfermagem, não sendo possível abranger todos os profissionais do setor de internação cirúrgica, pois no período possuíam cinco profissionais afastados por licença médica ou férias.

Para compor os dados, após a execução da TE, verificou-se a partir de busca ativa no período de seis meses se houve modificação ou aumento no número de registros de queda, tanto na ficha específica para notificação de EA encaminhadas ao NSP, como no livro de ordens e ocorrências do setor de internação cirúrgica.

Para fins de medida estatística, foi realizado o cálculo do índice de queda por paciente-dia e caracterizado o perfil das quedas notificadas. O índice de queda por paciente-dia foi obtido através da fórmula do Protocolo de Prevenção de Quedas do MS (2013): [ $\left(n^{\circ}\right.$ de eventos / $n^{\circ}$ de pacientedia)*1000].

Os dados encontrados foram organizados em tabelas para posterior análise descritiva dos resultados, em valor absoluto e percentual.

A pesquisa foi aprovada pelo Comitê de Ética em Pesquisa da Universidade Federal do Estado do Rio de Janeiro em 30 de outubro de 2014 com o Parecer $n^{\circ} 850.633$. 
A partir da busca ativa, verificou-se que no período de abril a agosto de 2015, anteriormente à aplicação da TE, houve o relato de uma queda, porém não notificada adequadamente na ficha específica de EA, demonstrado na Tabela 1.

Tabela 1 - Registro das quedas sofridas pelos pacientes internados no setor da clínica cirúrgica no período de abril de 2015 a fevereiro de 2016. Rio de Janeiro, RJ, Brasil, 2016

\begin{tabular}{lcc} 
Quedas & $\begin{array}{c}\text { Antes da aplicação da } \\
\text { Tecnologia }\end{array}$ & $\begin{array}{c}\text { Após aplicação da } \\
\text { Tecnologia }\end{array}$ \\
\hline Registro no Livro de Ordens e Ocorrências & 1 & 4 \\
\hline Notificação Adequada na ficha específica & 0 & 6 \\
\hline Total de Quedas & $\mathbf{1}$ & $\mathbf{1 0}$ \\
\hline Média de Paciente-dia Internado & 9,6 & 10 \\
\hline Média Índice Indicador de Queda & $33,3(3,33 \%)$ & $162,2(16,22 \%)$
\end{tabular}

Fonte: Livro de Ordens e Ocorrências e Instrumento de Notificação de EA da Instituição

Após a aplicação da TE, verificou-se, no período de setembro de 2015 a fevereiro de 2016, o registro de quatro quedas no livro de ordens e ocorrências e seis quedas notificadas adequadamente na ficha específica de notificação para EA. Ambas as ocorrências poderiam ser registradas tanto no livro de ordens e ocorrências como na ficha específica de notificação, porém ao realizarmos a análise destas não foi possível identificar nenhuma compatibilidade entre elas, permanecendo o quantitativo total de dez quedas no período estudado.

Destas dez quedas registradas após a execução da TE, três delas foram no mês de setembro, registradas apenas no livro de ordens e ocorrências. No mês de novembro foram registradas duas quedas, e outras quatro no mês de dezembro, ambas registradas adequadamente na ficha específica de notificação de EA. A última queda analisada foi no mês de fevereiro de 2016, registrada apenas no livro de ordens e ocorrências do setor de estudo.

Obtivemos uma média de 33,3 quedas por 1.000 paciente-dia (3,33\%) anteriormente à realização da TE e posteriormente 162,2 quedas por 1.000 paciente-dia (16,22\%). A elevação do índice após a execução da TE pode não significar necessariamente um aumento na ocorrência de quedas, mas pode indicar uma melhoria no sistema de busca ativa de notificações dos eventos, e também aumento do interesse dos profissionais em registrar a notificação.

No período do estudo após a execução da TE, foram notificadas adequadamente seis quedas na ficha específica de EA ao NSP, sendo possível verificar algumas caracterizações do seu perfil, conforme Tabela 2.

Tabela 2 - Caracterizações das quedas sofridas pelos pacientes internados no setor da clínica cirúrgica no período de setembro de 2015 a fevereiro de 2016. Rio de Janeiro, RJ, Brasil, 2016 (continua)

\begin{tabular}{lcc} 
Características & Número de Pacientes (n) & Percentual (\%) \\
\hline Sexo & & \\
\hline Masculino & 4 & 66,7 \\
\hline Feminino & 2 & 33,3 \\
\hline Idade & & \\
\hline Masculino & 65,7 anos & Média Total \\
\hline Feminino & 40 anos & 57,2 anos \\
\hline
\end{tabular}




\begin{tabular}{lll}
\hline Estado Mental & 4 & \\
\hline Orientado & 2 & 66,7 \\
\hline Desorientado & & 33,3 \\
\hline Local da Queda & 1 & 16,7 \\
\hline Queda do Leito & 1 & 16,7 \\
\hline Queda da Cadeira & 4 & 66,6 \\
\hline Queda da Própria Altura & & 66,7 \\
\hline Consequência da Queda & 4 & 33,3
\end{tabular}

Fonte: Instrumento de Notificação do Núcleo de Segurança do Paciente

Ao analisarmos o perfil das seis quedas notificadas adequadamente na ficha específica de EA, observa-se que quatro delas foram pacientes do sexo masculino e possuíam em média 57,2 anos de idade. Em duas das quedas, os pacientes encontravam-se desorientados no momento. Quatro quedas foram da própria altura, somando com uma do leito do paciente e uma queda da cadeira. Como consequências da queda, duas foram registradas com danos.

Observamos que as quedas notificadas na ficha específica não estavam preenchidas corretamente, havendo falhas na descrição do acidente e dados com informações incompletas.

\section{- DISCUSSÃO}

Avaliação do risco de queda deve ser feita no momento da admissão do paciente e diariamente com o emprego de escala adequada ao perfil de pacientes da instituição. As escalas de avaliação de risco de queda não são universais, e apresentam vantagens, mas também limitações operacionais e metodológicas. Permitem classificar o grau de risco que o paciente apresenta para cair, possibilitando orientar as intervenções necessárias para evitar a ocorrência de queda ${ }^{(2)}$.

Nesse contexto, identifica-se a importância da comunicação efetiva, em todos os níveis, sensibilizando os profissionais, evitando subnotificações de eventos, possibilitando a promoção da melhoria da qualidade e segurança do paciente nos serviços de saúde.

Diante disto, a construção do material em forma de slides e imagens visuais possui forte poder de representação, permitindo melhor compreensão e recordação do texto, favorecendo a memorização da imagem e da informação transmitida. Ajuda a ilustrar procedimentos e rotinas de enfermagem de forma atual e realista do cotidiano, garantindo significado. Os objetivos propostos devem seguir sequência lógica, com abordagem autoexplicativa e dinâmica, com linguagem clara, objetiva e coloquial, adequada às características da clientela, permitindo leitura de fácil compreensão ${ }^{(5)}$.

Baseado no número de notificações encontradas no banco de dados anteriormente à execução da $\mathrm{TE}$, a não utilização da ficha específica para notificação de EA talvez ocorresse pela falta de treinamentos ou desconhecimento do protocolo de quedas da instituição.

Após a execução da TE, houve adesão insatisfatória dos profissionais de enfermagem quanto ao registro das quedas na ficha específica de notificação de EA. No último mês da busca ativa, fevereiro de 2016, verificou-se uma queda do paciente no livro de ordens e ocorrências apenas, não tendo seu registro realizado de forma adequada na ficha específica de notificação de EA. Destacamos a importância da educação continuada para a efetiva realização do registro de queda na ficha específica de notificação de EA e seu correto preenchimento.

Para contribuição deste resultado, infere-se que os profissionais possam se sentir desestimulados e desacreditados frente ao próprio NSP da instituição. Outros fatores que podem influenciar são a falta de recursos humanos, cultura de segurança e fatores estruturais da instituição. 
Sendo assim, as práticas educativas têm importante papel nas ações de educação em saúde, orientações e estímulo à equipe, para buscar melhorias dos processos, minimizar os riscos e fortalecer práticas seguras. Além da realização de educação continuada, é preciso proporcionar condições de trabalho que possibilitem o compartilhamento de responsabilidades e gestão aberta a opiniões, para que toda a equipe tenha como ideal a segurança do paciente ${ }^{(6-7)}$.

Também é preciso enfrentar e superar posturas de resistência de alguns profissionais que, por desconhecimento ou pouca valorização da importância, não aderem à proposta imediatamente. Existe ainda a necessidade de avaliar a eficácia e o impacto de ações educativas de modo sistematizado, possibilitando a identificação dos fatores que restringem ou contribuem para o melhor desempenho dos profissionais ${ }^{(8)}$.

Para as notificações serem realizados integralmente, necessita-se da colaboração de toda a equipe de enfermagem, possibilitando assim que compreendam sua importância e colaborem para a sua execução de forma efetiva. Muitas vezes as notificações são deficientes, fato que pode estar relacionado ao número reduzido de trabalhadores e à falta de tempo para registrar a assistência prestada e ocorrência de EA. Porém, existem outros aspectos que podem ser limitantes, como por exemplo, a escassez de recursos físicos, estruturais, materiais, dificuldades no acesso ao computador e no seu manejo, prática educativa monótona, desestimulante e repetitiva ${ }^{(9-10)}$.

É necessária a implantação dos indicadores de segurança do paciente, pois subsidiam o monitoramento de EA e erros ocorridos durante internações. Estes indicadores são instrumentos valiosos para auxiliar na identificação de áreas que precisam ser mais bem estudadas, com vistas à melhoria da qualidade dos cuidados prestados aos pacientes. Podendo ser utilizado os dados do prontuário do paciente e o instrumento de notificação de quedas ${ }^{(2)}$.

O cálculo da incidência de queda é realizado com o número de quedas total dividido pelo número de pacientes-dia, vezes 1000. Este indicador também pode ser monitorado utilizando um diagrama de controle para a construção da série histórica do evento, e auxílio para estabelecer metas e parâmetros de avaliação ${ }^{(2)}$. Nesta pesquisa obteve-se, após a TE, 162,2 quedas por 1.000 paciente-dia.

O índice desejado deve ser baixo, pois estes estão associados a maior adoção de medidas preventivas para diminuição da incidência de quedas. Em hospitais de países desenvolvidos, a taxa de queda de pacientes variou entre três a cinco quedas por 1.000 pacientes-dia. Um estudo realizado em São Paulo apresentou uma taxa de queda de 1,45 por 1.000 pacientes-dia, considerada reduzida devido à implementação de um protocolo de gerenciamento de quedas ${ }^{(2)}$.

Um hospital de grande porte no Rio Grande do Sul implementou os indicadores de qualidade assistencial de incidências de quedas estabelecendo a meta de $\leq$ duas por 1.000 paciente-dia/mês. Outros estudos mostram variações no índice de quedas que vão de 1,4 a 13,0 quedas por 1.000 pacientes-dia. Estudo mais recente realizado no setor de cardiologia de um hospital em São Paulo registrou a incidência de 2,04 quedas por 1.000 paciente-dia ${ }^{(11-12)}$.

Das seis quedas notificadas na ficha específica de EA ao NSP, foi possível identificar o perfil destas, como pacientes do sexo masculino que representaram $66,7 \%$ que sofreram quedas e do sexo feminino $33,3 \%$. Corroborando os dados com outros estudos que identificam uma maior prevalência de quedas no sexo masculino, incluindo um que ressalta predominância no sexo masculino em cerca de $59,7 \%^{(12-}$ 13).

A média de idade dos pacientes que sofreram queda foi de 57,2 anos, sendo 65,7 anos entre os homens e 40 anos entre as mulheres, divergindo com outros estudos. Estes apontam os idosos como sujeitos com maiores índices de quedas, devido ao processo natural e gradual do envelhecimento que proporciona mudanças físicas, como perda de força, diminuição da coordenação, alterações cognitivas e procedimentos médicos, além do uso concomitante de poli fármacos que acarretam maior vulnerabilidade para quedas ${ }^{(13)}$. Inclui-se aqui outro estudo mostrando que $53 \%$ das quedas ocorreram em pacientes com mais de 65 anos $^{(14)}$.

Contudo, nos setores de neuroclínica e neurocirurgia o risco de queda é maior e independe da idade, ocorrendo duas vezes mais entre pacientes da neurologia. Estes pacientes possuem fatores de risco como alterações no nível de consciência, mobilidade prejudicada, hipotensão ortostática, 
distúrbios vesicais ou intestinais, déficits sensoriais e história prévia de quedas. Também demonstraram a incidência de danos provocados por quedas de $28 \%$ nas unidades de neuroclínica e neurocirurgia; $33,6 \%$ de danos em outras unidades de internação ${ }^{(14)}$.

Em síntese, quedas mais frequentes estão presentes nas unidades com concentração de pacientes idosos, na neurologia e na reabilitação. Havendo também relatos de maior ocorrência de queda em unidades de cuidado de longa permanência ${ }^{(2)}$, não sendo característica do setor cirúrgico estudado, pois havia curta permanência e alta rotatividade de pacientes.

As quedas aconteceram, em sua maioria, com pacientes que não apresentavam alteração do estado mental, $66,7 \%$ dos pacientes estavam lúcidos e orientados no momento da queda. Estudos apontam que apenas 19,6\% dos pacientes que sofreram queda apresentavam alteração no nível de consciência, sendo a maioria no período pós-operatório ${ }^{(14)}$.

Esta pesquisa corrobora com o fato de que pacientes orientados são mais susceptíveis à queda, quando fora do espaço da cama, pois não solicitaram auxílio para executar determinadas atividades como higiene pessoal, tentar se levantar do leito ou ao retornar à cama sozinho, subindo pelas grades da cama muitas vezes ${ }^{(15-16)}$.

No momento da queda, $16,7 \%$ dos pacientes encontravam-se no leito, $16,7 \%$ na poltrona e $66,6 \%$ em pé na própria altura, dados que se assemelham a pesquisa que aponta que $64,2 \%$ das quedas acontecem da própria altura ${ }^{(12)}$.

Ao analisar as consequências das quedas, cerca de 33,3\% causaram danos aos pacientes, sendo classificados como ferimentos corto-contusos em membros inferiores com necessidade de sutura, e associadas ao ambiente físico devido ao equipamento danificado, no caso as travas de freio do leito. Pesquisas apontam as quedas como causadoras de danos em $30 \%$ a $50 \%$ dos casos, sendo que $6 \%$ a $44 \%$ desses pacientes sofrem danos de natureza grave, como traumas teciduais de diferentes intensidades, retirada não programada ou desconexão de diferentes artefatos terapêuticos, alterações emocionais como ansiedade, depressão e medo de cair novamente. Estas ocorrências resultam em piora das condições clínicas, óbito, aumento no tempo da internação, aumento no custo do tratamento e a descrença do paciente em relação aos serviços de enfermagem ${ }^{(2,17)}$.

Geralmente a queda está associada a fatores vinculados tanto ao indivíduo como ao ambiente físico. Entre os fatores vinculados ao paciente, destacam-se: idade avançada, história recente de queda, redução da mobilidade, incontinência urinária, uso de medicamentos e hipotensão postural. Com relação aos fatores ambientais e organizacionais, podem ser citados: pisos desnivelados, objetos largados no chão, altura inadequada da cadeira, insuficiência e inadequação dos recursos humanos ${ }^{(2)}$.

\section{CONCLUSÕES}

O estudo aponta que a TE pode ser adotada como estratégia para aumentar as notificações de EA.

A cultura de segurança ainda é algo a ser difundido na instituição referida, apontando-se como uma limitação da pesquisa. A notificação da queda foi vista como um problema, pois não há uma rotina de prevenção de queda constituída de forma satisfatória e nem formas de capacitação continuada, já que se observou falha na notificação da queda e prováveis casos subnotificados. É também imprescindível a implantação de uma escala de avaliação do risco de queda na rotina da instituição.

A pesquisa apontou como limitação o período de coleta de dados das notificações de queda, e o baixo número de quedas registradas neste período, indicando a necessidade da continuação do estudo no mesmo cenário.

\section{REFERÊNCIAS}

1. Hemesath MP, dos Santos HB, Torelly EMS, Barbosa AS, de Magalhães AMM. Estratégias educativas para melhorar adesão à identificação do paciente. Rev. Gaúcha Enferm. [Internet] 2015;36(4) [acesso em 10 fev 2016]. Disponível: http://dx.doi.org/10.1590/1983-1447.2015.04.54289. 
2. Ministério da Saúde (BR). Agencia Nacional de Vigilância Sanitária. Fiocruz. Protocolo de Prevenção de Quedas. Proqualis. [Internet] 2013 [acesso em 10 mai 2015]. Disponível: http://www.saude.mt.gov.br/upload/controleinfeccoes/pasta12/protocolos_cp_n6_2013_prevencao.pdf.

3. Ministério da Saúde (BR). Portaria n. 529, de 1 de abril de 2013. Dispõe sobre o Programa Nacional de Segurança do Paciente. [Internet] Diário Oficial da República Federativa do Brasil, Brasília, 2 abr. 2013 [acesso em 10 dez 2015]. Disponível: http://bvsms.saude.gov.br/bvs/saudelegis/gm/2013/prt0529_01_04_2013.html.

4. Nietsche EA, de Lima MGR, Rodrigues MGS, Teixeira JA, de Oliveira BNB, Motta CA, et al. Tecnologias inovadoras do cuidado em enfermagem. Rev Enferm UFSM. [Internet] 2012;2(1) [acesso em 6 ago 2015]. Disponível: https:// periodicos.ufsm.br/reufsm/article/view/3591.

5. Ministério da Saúde (BR). Fundação Oswaldo Cruz. Educação a Distância. Elaboração de material didático impresso para programas de formação a distância: orientações aos autores. [Internet] 2005 [acesso em 10 fev 2016]. Disponível: http://www.ufrgs.br/nucleoead/documentos/ENSPMaterial.pdf.

6. Costa VT, Meirelles BHS, Erdmann AL. Melhores práticas do enfermeiro gestor no gerenciamento de risco. Rev. Latino-Am. Enfermagem. [Internet] 2013;21(5) [acesso em 21 ago 2015]. Disponível: http://dx.doi.org/10.1590/ S0104-11692013000500021.

7. Carneiro FS, Bezerra ALQ, de Camargo e Silva AEB,de Souza LP, Paranaguá TTB, Branquinho NCSS. Evento adverso na clínica cirúrgica em um hospital universitário: instrumento de avaliação da qualidade. Rev. enferm. UERJ. [Internet] 2011;19(2) [acesso em 21 ago 2015]. Disponível: http://www.facenf.uerj.br/v19n2/v19n2a06.pdf.

8. Mira VL, Ortiz DCF, Follador NN, Ferrari CRS, Oliveira LFMN, Silva JAM, et al. Avaliação da eficácia de um treinamento de profissionais de enfermagem: estudo correlacional. Online Braz J Nurs. [Internet] 2012;11(3) [acesso em 2 ago 2015]. Disponível: http://www.objnursing.uff.br/index.php/nursing/rt/printerFriendly/3652/ html.

9. Silva JA, Grossi ACM, Haddad MCL, Marcon SS. Avaliação da qualidade das anotações de enfermagem em unidade semi-intensiva. Esc. Anna Nery. [Internet] 2012;16(3) [acesso em 28 out 2015]. Disponível: http://dx.doi. org/10.1590/S1414-81452012000300021.

10. Fonseca LMM, Leite AM, de Mello DF, Silva MAI, de Lima RAG, Scochi CGS. Tecnologia educacional em saúde: contribuições para a enfermagem pediátrica e neonatal. Esc. Anna Nery. [Internet] 2011;15(1) [acesso em 10 ago 2015]. Disponível: http://dx.doi.org/10.1590/S1414-81452011000100027.

11. Correa AD, Marques IAB, Martinez MC, Laurino PS, Leão ER, Chimentão DMN. Implantação de um protocolo para gerenciamento de quedas em hospital: resultados de quatro anos de seguimento. Rev. esc. enferm. USP. [Internet] 2012;46(1) [acesso em 19 set 2015]. Disponível: http://dx.doi.org/10.1590/S0080-62342012000100009.

12. Meneguin S, Ayres JA, Bueno GH. Caracterização das quedas de pacientes em hospital especializado em cardiologia. Rev Enferm UFSM. [Internet] 2014;4(4) [acesso em 10 ago 2015]. Disponível: http://dx.doi. org/10.5902/2179769213554.

13. Dunne TJ, Gaboury I, Ashe MC. Falls in hospital increase length of stay regardless of degree of harm. J Eval Clin Pract. [Internet] 2014;20(4) [acesso em 28 out 2014]. Disponível: http//dx.doi.org/10.1111/jep.12144.

14. Diccini S, de Pinho PG, da Silva FO. Avaliação de risco e incidência de queda em pacientes neurocirúrgicos. Rev. Latino-Am. Enfermagem. [Internet] 2008;16(4) [acesso em 10 set 2015]. Disponível: http://dx.doi.org/10.1590/ S0104-11692008000400016.

15. Hignett S, Sands G, Griffiths P. In-patient falls: what can we learn from incidente reports?. Age and Ageing. [Internet] 2013;42(4) [acesso em21 jan 2015]. Disponível: http://dx.doi.org/10.1093/ageing/aft058.

16. de Paiva MCMS, de Paiva SAR, Berti HW, Campana AO. Caracterização das quedas de pacientes segundo notificação em boletins de eventos adversos. Rev. esc. enferm. USP. [Internet] 2010;44(1) [acesso em 22 jan 2015$].$ Disponível: http://dx.doi.org/10.1590/S0080-62342010000100019.

17. Costa SGRF, Monteiro DR, Hemesath MP, Almeida MA. Caracterização das quedas do leito sofridas por pacientes internados em um hospital universitário. Rev. Gaúcha Enferm. [Internet] 2011;32(4) [acesso em 22 jan 2015]. Disponível: http://hdl.handle.net/10183/96283. 